\title{
Isoperimetric characterization of the incenter of a triangle
}

\author{
Jun O'Hara
}

Jun O'Hara obtained his Ph.D. from the University of Tokyo in 1991. He is now Associate Professor at the Tokyo Metropolitan University. His main mathematical interests are in knot energies and the Möbius geometry of curves and surfaces.

\section{Introduction}

Recently Katsuyuki Shibata introduced a new kind of center of a triangle, which he calls the illuminating center ([3]). It is a point that maximizes the total brightness of a triangular park $\Omega$ obtained by a light source on that point, namely, a point that maximizes $V_{0}(x)=$ $\int_{\Omega}|x-y|^{-2} d \mu(y)$, where $\mu$ is the standard Lesbegue measure of $\mathbb{R}^{2}$. Unfortunately, $V_{0}(x)$ is not well-defined; it diverges for any point in $\Omega$. In order to produce a well-defined potential, Shibata used the cut-off of the divergence of the integrand.

In [2] the author introduced the renormalization of $\int_{\Omega}|x-y|^{\alpha-m} d \mu(y)$ (which is called the Riesz potential when $0<\alpha<m$ ) of a compact subset $\Omega$ in $\mathbb{R}^{m}$ which is a closure of an open set for $\alpha \leq 0$ to obtain a one-parameter family of (renormalized) potentials $V_{\Omega}^{(\alpha)}$, and studied the points where the extremal values of $V_{\Omega}^{(\alpha)}$ are attained, which we call the $r^{\alpha-m}$-centers of $\Omega$. The notion of $r^{\alpha-m}$-centers includes not only Shibata's illuminating center of a planar domain as an $r^{-2}$-center, but also the center of mass of any compact set $\Omega \subset \mathbb{R}^{m}$ as $r^{2}$-center. This is because the center of mass $x_{G}$ is given by

Clark Kimberling listet auf seiner Web-Seite Encyclopedia of Triangle Centers inzwischen weit über 5000 Dreieckszentren auf. Dort ist z.B. X(1) der Inkreismittelpunkt, $X(2)$ der Schwerpunkt, oder $X(54)$ der Kosnita-Punkt eines Dreiecks. Zahlreiche dieser Zentren lassen sich auf unterschiedliche Weise charakterisieren. In der vorliegenden Arbeit wird gezeigt, dass der Inkreismittelpunkt gleichzeitig eine gewisse Funktion minimiert: Dazu betrachtet man das Dreieck als Grundfläche einer Pyramide mit Spitze $p$. Aus deren Volumen und Oberfläche bildet man sodann einen geeigneten skaleninvarianten von $p$ abhängigen Quotienten. Minimiert man die so definierte Funktion so fällt die Projektion des optimalen Punktes $p$ auf die Grundfläche just in den Inkreismittelpunkt des Dreicks. 
$x_{G}=\int_{\Omega} y d \mu(y) / \int_{\Omega} 1 d \mu(y)$, or equivalently by $\int_{\Omega}\left(x_{G}-y\right) d \mu(y)=0$, which implies that it can be characterized as a unique critical point of the map $V_{\Omega}^{(m+2)}: \mathbb{R}^{m} \ni x \mapsto$ $\int_{\Omega}|x-y|^{2} d \mu(y) \in \mathbb{R}$.

Shibata announced ${ }^{1}$ a theorem that an $r^{a}$-center of a non-obtuse triangle approaches the circumcenter as $a$ goes to $+\infty$ and to the incenter as $a$ goes to $-\infty$. The proof with more generality is given in [2]. Thus, we can give interpretations of the barycenter, circumcenter, and incenter of a triangle as points that optimize a kind of potential and the limits of them.

The motivation of the theorem in this note comes from the same philosophy; to express a center as a point that optimizes a kind of potential. Our potential in this note is the ratio of the volume of the cone over a given triangle $\Omega$ and the area of its boundary, with the former being squared and the latter cubed to make the ratio scale invariant. Then, the image of the regular projection of a vertex of a cone that optimizes this ratio is nothing but the incenter.

\section{Cone isoperimetric center}

Let $\Omega$ be a compact set which is a closure of an open subset of $\mathbb{R}^{2}$ with a piecewise $C^{1}$ boundary $\partial \Omega$. We assume that $\mathbb{R}^{2}$ is embedded in $\mathbb{R}^{3}$ in a standard way; $\mathbb{R}^{2}=$ $\left\{\left(x_{1}, x_{2}, 0\right) \in \mathbb{R}^{3} \mid x_{i} \in \mathbb{R}\right\}$. Let $\Pi_{h}$ denote a level plane in $\mathbb{R}^{3}$ with height $h>0$, $\Pi_{h}=\left\{x_{3}=h\right\}$, and $C_{p}$ a cone over $\Omega$ with vertex $p \in \Pi_{h}, C_{p}=\{t x+(1-t) p \mid x \in$ $\Omega, 0 \leq t \leq 1\}$. Let $\pi: \mathbb{R}^{3} \rightarrow \mathbb{R}^{2}$ be the regular projection.

\section{Definition 2.1.}

(1) Let $p_{h}$ be a point in $\Pi_{h}$ where the minimum value of a function $\Pi_{h} \ni p \mapsto$ Area $\left(\partial C_{p}\right)$ is attained. We call $\pi\left(p_{h}\right)$ a cone isoperimetric center of $\Omega$ of height $h$.

(2) Let $p$ be a point in $\mathbb{R}_{+}^{3}=\left\{x_{3}>0\right\}$ that gives the minimum value of a function

$$
f(p)=\frac{\left(\operatorname{Area}\left(\partial C_{p}\right)\right)^{3}}{\left(\operatorname{Vol}\left(C_{p}\right)\right)^{2}} .
$$

We call $C_{p}$ an isoperimetrically optimal cone and $\pi(p)$ a cone isoperimetric center of $\Omega$.

Lemma 2.2. Let $\triangle A B C$ be a triangle. Then there exists a cone isoperimetric center of height $h$ for any $h>0$.

Proof. Let $S$ be the area, and $a, b$, and $c$ the lengths of the edges $B C, C A$, and $A B$, respectively. Fix $h>0$. Let $P \in \Pi_{h}$ be a point and $D=\pi(P)$. Let $u, v$, and $w$ be the distances with signs between $D$ and the lines $\overline{B C}, \overline{C A}$, and $\overline{A B}$, respectively. The signs of $u, v$, and $w$ are given as follows. We put $u>0$ if $D$ and $A$ are in the same half-plane cut out by the line $\overline{B C}$. Remark that the position of $D$ is determined uniquely by $u$ and $v$.

1. at 2010 Autumn Meetings of the Mathematical Society of Japan 
Then the area of the triangle $\triangle A B C$ is given by $S=\frac{1}{2}(a u+b v+c w)$, and the area of the boundary of the cone is given by

$$
\text { Area }\left(\partial C_{P}\right)=S+\frac{1}{2}\left(a \sqrt{u^{2}+h^{2}}+b \sqrt{v^{2}+h^{2}}+c \sqrt{w^{2}+h^{2}}\right) .
$$

Let the right-hand side of (1) be denoted by $\psi(D)$. Then, it takes the value $S+\frac{1}{2}(a+b+$ c) $\sqrt{r^{2}+h^{2}}$ at the incenter $I$, where $r$ is the radius of the inscribed circle. Put

$$
\rho=\frac{a+b+c}{\min \{a, b, c\}} \sqrt{r^{2}+h^{2}} .
$$

Let $\bar{N}_{\rho}(\overline{B C})$ be the set of points so that the distance to the line $\overline{B C}$ is not greater than $\rho$, namely, a closed strip with central axis $\overline{B C}$ which is $2 \rho$ wide. Two other strips, $\bar{N}_{\rho}(\overline{C A})$ and $\bar{N}_{\rho}(\overline{A B})$, can be defined similarly. Put $K=\bar{N}_{\rho}(\overline{B C}) \cap \bar{N}_{\rho}(\overline{C A}) \cap \bar{N}_{\rho}(\overline{A B})$. Then $K$ is a compact set containing $I$.

Suppose $D \notin K$. Then at least one of $|u|,|v|$, and $|w|$ is greater than $\rho$. Therefore,

$$
\psi(D)>S+\frac{1}{2} \min \{a, b, c\} \sqrt{\rho^{2}+h^{2}}>S+\frac{1}{2} \min \{a, b, c\} \rho=\psi(I),
$$

which implies $\inf _{D^{\prime} \in K} \psi\left(D^{\prime}\right)=\inf _{D^{\prime \prime} \in \mathbb{R}^{2}} \psi\left(D^{\prime \prime}\right)$. Since $\psi$ is continuous and $K$ is compact, there is a point $D \in K$ where $\inf _{D^{\prime} \in K} \psi\left(D^{\prime}\right)$ is attained.

It follows that $\inf _{D^{\prime \prime} \in \mathbb{R}^{2}} \psi\left(D^{\prime \prime}\right)$ is also attained at $D$.

Theorem 2.3. Let $\triangle A B C$ be a triangle. The cone isoperimetric center of height $h$ coincides with the incenter for any $h>0$. The height of the isoperimetrically optimal cone is $2 \sqrt{2}$ times the radius of the inscribed circle.

Proof. (1) Let us use the same notation as in Lemma 2.2.

Let $D_{h}$ be a cone isoperimetric center of $\triangle A B C$ of height $h$, and $u_{h}, v_{h}$, and $w_{h}$ be the signed distances between $D_{h}$ and the lines $\overline{B C}, \overline{C A}$, and $\overline{A B}$, respectively. Then the pair $\left(u_{h}, v_{h}\right)$ minimizes a function

$$
F(u, v)=a \sqrt{u^{2}+h^{2}}+b \sqrt{v^{2}+h^{2}}+c \sqrt{\left(\frac{2 S-a u-b v}{c}\right)^{2}+h^{2}} .
$$

Therefore, when $(u, v, w)=\left(u_{h}, v_{h}, w_{h}\right)$ we have

$$
\begin{aligned}
& F_{u}(u, v)=\frac{a u}{\sqrt{u^{2}+h^{2}}}+\frac{c w}{\sqrt{w^{2}+h^{2}}} \cdot\left(-\frac{a}{c}\right)=0, \\
& F_{v}(u, v)=\frac{b v}{\sqrt{v^{2}+h^{2}}}+\frac{c w}{\sqrt{w^{2}+h^{2}}} \cdot\left(-\frac{b}{c}\right)=0,
\end{aligned}
$$

which implies

$$
\frac{u}{\sqrt{u^{2}+h^{2}}}=\frac{v}{\sqrt{v^{2}+h^{2}}}=\frac{w}{\sqrt{w^{2}+h^{2}}} .
$$


Remark that the above holds only when $u, v$, and $w$ are all positive, implying that $D_{h}$ is in the interior of $\triangle A B C$. The equation (2) means that three angles between the $x y$-plane and three planes through $P A B, P B C$, and $P C A$ are all equal. Therefore, each pair of the three planes is symmetric in a plane which is orthogonal to the $x y$-plane and contains the intersection line of the pair. These three symmetries show that the three lines $D_{h} A$, $D_{h} B$, and $D_{h} C$, which are the intersections of the $x y$-plane and the three planes of the symmetries, are the angle bisectors of $\angle A, \angle B$, and $\angle C$, respectively. It follows that $D_{h}$ coincides with the incenter of $\triangle A B C$.

(2) The second statement follows from elementary calculus. Let $r$ be the radius of the inscribed circle. Put $P_{h}=\pi^{-1}\left(D_{h}\right) \cap \Pi_{h}$, then

$$
\operatorname{Area}\left(\partial C_{P_{h}}\right)=S+\frac{1}{2}(a+b+c) r \sqrt{1+\left(\frac{h}{r}\right)^{2}}=S\left(1+\sqrt{1+\left(\frac{h}{r}\right)^{2}}\right) \text {. }
$$

As $\operatorname{Vol}\left(C_{P_{h}}\right)=\frac{1}{3} S h$,

$$
f\left(P_{h}\right)=\frac{\left(\operatorname{Area}\left(\partial C_{P_{h}}\right)\right)^{3}}{\left(\operatorname{Vol}\left(C_{P_{h}}\right)\right)^{2}}=9 S \frac{\left(1+\sqrt{1+\left(\frac{h}{r}\right)^{2}}\right)^{3}}{h^{2}}=\frac{9 S}{r^{2}} \cdot \frac{\left(1+\sqrt{1+\left(\frac{h}{r}\right)^{2}}\right)^{3}}{\left(\frac{h}{r}\right)^{2}} .
$$

Since $\varphi(t)=\frac{\left(1+\sqrt{1+t^{2}}\right)^{3}}{t^{2}}(t>0)$ takes the minimum at $t=2 \sqrt{2}$, it completes the proof.

Remark 2.4. The above theorem means that the cone isoperimetric center of height $h$ is identically the same for any $h>0$ and that it coincides with the limit of $r^{a}$-center as $a$ goes to $-\infty$ for triangles. But it does not hold in general as an example below shows.

Let us call a point an asymptotic $r^{-\infty}$-center of $\Omega$ if it is the limit of a convergent sequence of $r^{a_{i}}$-centers with $a_{i} \rightarrow-\infty$ as $i \rightarrow+\infty$. We showed in [2] that an asymptotic $r^{-\infty}$ center is a max-min point of $\Omega$, by which we mean a point that gives the supremum of a $\operatorname{map} \mathbb{R}^{2} \ni x \mapsto \min _{y \in \overline{\Omega^{c}}}|y-x| \in \mathbb{R}$, where $\overline{\Omega^{c}}$ denotes the closure of the complement of $\Omega$. We remark that an $r^{a}$-center $(a \leq-2)$ and a max-min point are not necessarily unique. To see this, it is enough to consider a disjoint union of two rectangles, say, $\Omega^{\prime}=\{(\xi, \eta) \mid$ $1 \leq|\xi| \leq 2,|\eta| \leq 2\}$.

Let $\Omega$ be a trapezoid given by $\Omega=\left\{(\xi, \eta)|0 \leq \xi \leq 2,| \eta \mid \leq 1+\frac{1}{2} \xi\right\}$. It is easy to see that a cone isoperimetric center of height $h$ is on the $\xi$-axis for any $h$. Let it be given by $\left(\xi_{h}, 0\right)$. Numerical experiments show that $\xi_{1} \sim 0.9169, \xi_{2} \sim 0.9079, \xi_{3} \sim 0.9045$, and $\xi_{4} \sim 0.9031$, and the minimum of the ratio $f$ is attained at $h \sim 3.250$ when $\xi_{h} \sim 0.90405$. On the other hand, an asymptotic $r^{-\infty}$-center is $(1,0)$. This is because the set of max-min points is $\left\{(1, \eta)|| \eta \mid \leq \frac{3}{2}-\frac{\sqrt{5}}{2}\right\}$ whereas any $r^{a}$-center is contained in $\left\{(\xi, 0) \mid 1 \leq \xi \leq \frac{7}{4}\right\}$ for any $a$ by the symmetry argument (based on the moving plane method [1]) explained in [2], and the point $(1,0)$ is the unique intersection point of these sets. 


\section{References}

[1] Gidas, B.; Ni, W.M.; Nirenberg, L.: Symmetry and related properties via the maximum principle. Comm Math. Phys. 68 (1979), 209-243.

[2] O’Hara, J.: Renormalization of potentials and generalized centers. To appear in Adv. Appl. Math.

[3] Shibata, K.: Where should a streetlight be placed in a triangle-shaped park? Elementary integro-differential geometric optics.

Available at http://www1.rsp.fukuoka-u.ac.jp/kototoi/shibataaleph-sjs.pdf

Jun O'Hara

Department of Mathematics and Information Sciences

Tokyo Metropolitan University

1-1 Minami-Ohsawa, Hachiouji-Shi

Tokyo 192-0397, Japan

e-mail: ohara@tmu.ac.jp 